\title{
Effets globaux d'une agglomération sur la typologie ichtyenne d'un fleuve : cas de la Garonne à Toulouse (France)
}

\author{
R. A. Hutagalung 1 \\ P. $\operatorname{Lim}^{1}$ \\ A. Belaud ${ }^{1}$ \\ T. Lagarrigue ${ }^{1}$
}

Mots clés : Garonne, peuplement ichtyques, échantillonnage ponctuel d'abondance, échantillonnage par filets, typologie, facteurs anthropiques.

Dans le but d'étudier la composition de l'ichtyofaune et les grandes tendances des relations poissons-habitats dans les milieux anthropisés, 4 secteurs d'un tronçon de $50 \mathrm{~km}$ de la Garonne ont été suivis en hiver 1994 et été 1995 . Les écoulements sont lotiques et correspondent à la Zone à Barbeau pour les secteurs amont et aval de Toulouse. Dans la traversée de l'agglomération, la Garonne est divisée en bras supérieur et bras inférieur et les écoulements des deux secteurs correspondent à la Zone à Brême. Le secteur du bras inférieur et le secteur aval Toulouse sont soumis à de fortes pressions anthropiques.

Les techniques d'échantillonnages utilisées sont les pêches électriques (méthode EPA) dans les secteurs lotiques et les pêches aux filets (EPF) dans les secteurs artificialisés à écoulement lentique.

27 espèces appartenant à 10 familles, à dominance de Cyprinidae, ont été recensées. Le peuplement est dominé par le barbeau (Barbus barbus L.), le goujon (Gobio gobio L.) et le chevaine (Leuciscus cephalus L.), en milieu lotique et par le gardon (Rutilus rutilus L.), la brême (Abramis brama L.) et l'ablette (Alburnus alburnus L.), en milieu lentique. Pour les 2 secteurs les plus soumis aux actions humaines, les différences par rapport aux secteurs moins anthropisés portent sur des espèces occasionnelles ou accidentelles. Ces différences sont plus marquées en milieu lentique. En effet, la richesse spécifique et l'abondance sont nettement plus élevées dans le bras inférieur (malgré les fortes actions humaines) que dans le bras supérieur où l'eau est pourtant de meilleure qualité.

Les relations poissons-habitats en milieu lotique sont peu évidentes pour la majorité des espèces. En milieu lentique, la préférence d'habitat dans le bras supérieur est orientée vers les enrochements et la végétation aquatique. Les relations poissons-habitats sont perturbées dans le bras inférieur, morphodynamiquement comparable au bras supérieur, mais soumis à de fortes actions anthropiques.

Influence of anthropogenic disturbance on freswater fish communities : case of the Garonne River in Toulouse (France)

Keywords : Garonne, fish community, electro-fishing of spot samples, gill-nets sampling, typology, anthropogenic disturbances.

A study of the fish community and its relation with habitat in an anthropogenically modified part of the Garonne River was conducted in 1994-1995. Four sectors in a segment of $50 \mathrm{kms}$ were surveyed. These sectors consisted of two types of channel forms : a lotic type situated upstream and downstream (corresponding to the barbel zone), and a lentic type in Toulouse city which is divided in two arms, superior arm and inferior arm (corresponding to the bream zone). The downstream and inferior arm sectors were subject to anthropogenic disturbances, while the two others were less disturbed.

According to the channel forms, fish were sampled using two different methods ; regular abundance sampling using electro-fishing of spot samples (EPA) for the lotic sectors and gill-nets (EPF) for the lentic sectors.

Twenty- seven species belonging to 10 families (dominated by Cyprinidae) were caught in winter 1994 and summer 1995. The lotic- sectors were dominated by barbel (Barbus barbus L.), gudgeon (Gobio gobio L.) and chub (Leuciscus cephalus L.) and the lentics sectors by roach (Rutilus rutilus L.), bream (Abramis brama L.) and bleak (Alburnus alburnus $\mathrm{L}$.). The effect of anthropogenic disturbances was evaluated by comparing the secondary and rare species in the different sectors studied. This difference was more obvious by comparing the two sectors of the lentic channel forms (the two arms of the Garonne in Toulouse). The inferior arm (especially subjected to industrial pollution) had higher species richness and abundance than the superior arm (unpolluted).

For the majority of species, the fish-habitat relationships in the lotic sectors were not obvious. In the lentic sectors, the habitat preference in the superior arm (unpolluted) was oriented to the sites with rocky bank and aquatic vegetation. This relation was

\footnotetext{
1. Laboratoire Ingénierie Agronomique, Equipe Environnement Aquatique et Aquaculture, INP-ENSA, 145 Avenue de Muret, F-31076 Toulouse
} Cedex, France. 


\section{Introduction}

Les transferts de matière et d'énergie en milieu fluvial s'effectuent principalement dans le sens amontaval (Vannote et al. 1980), sans négliger les échanges latéraux et verticaux qui prennent de plus en plus d'importance vers l'aval (Décamps \& Naiman 1989, Schiemer \& Zalewski 1992) et conditionnent la qualité des eaux et des habitats.

Les événements hydrauliques saisonniers modulent ce continuum qui se conserve cependant au cours du temps. En revanche, les barrages et autres activités humaines créent de brusques changements assimilables à des discontinuités de l'évolution longitudinale [«serial discontinuity concept» de Ward \& Stanford (1983)]. La traversée d'une grande agglomération, avec ses divers aménagements physiques, ses rejets domestiques et industriels, est une zone propice à l'étude de ces discontinuités.

Il est a priori admis que les peuplements ichtyens soumis à une discontinuité, tant des habitats physiques que de la qualité des eaux, doivent réagir au niveau de leur composition spécifique ou de leur comportement de sélection d'habitat. Mais cette hypothèse n'a été jusqu'à présent que très rarement expérimentée sur les grands fleuves (Oberdorff et al. 1993).

La majorité des actions humaines s'exerce sur les cours moyen et aval des fleuves, zones dominées par les Cyprinidae, dont les exigences écologiques et les stratégies d'occupation de l'habitat sont plus complexes et moins connues que celles des Salmonidae (Baras \& Cherry 1990, Pouilly 1994). Les avancées récentes sur l'écologie des Cyprinidae (Philippart \& Baras 1989, Philippart et al. 1990, Bouchard 1996, Mann 1996) permettent néanmoins d'aborder les incidences des perturbations du milieu sur les poissons.

La présente étude a été réalisée sur un segment de $50 \mathrm{~km}$ de Garonne qui, dans son ensemble, est peu aménagé physiquement (Décamps \& Naiman 1989). Le tronçon étudié est de type lotique (pente moyenne $1,3 \%$ ) à l'exception de la traversée de la ville de Toulouse (400 000 habitants) où le fleuve est artificiellement divisé, sur $4 \mathrm{~km}$, en 2 bras à caractère lentique. L'un de ces bras reçoit des rejets industriels, l'autre non. Les rejets domestiques sont déversés, après traitement, en aval de la ville.

Cette approche globale des effets de diverses actions humaines vise à comparer directement, d'une part l'amont et l'aval d'une agglomération et, d'autre part, les 2 deux bras de la Garonne dans l'agglomération toulousaine. La recherche entreprise a porté d'une part, sur la composition de l'ichtyofaune en tenant compte du type de milieu lotique ou lentique et d'autre part, sur les grandes tendances des relations poissons-habitats.

\section{Zone d'étude}

Le tronçon de Garonne étudié (Fig. 1) recouvre 4 secteurs : 1 = amont Toulouse, 2 = Toulouse bras supérieur (non pollué), $3=$ Toulouse bras inférieur (rejets industriels) et $4=$ aval Toulouse. Au sein de chaque secteur, trois ou quatre stations de mesure ont été sélectionnées selon des critères morphodynamiques, de manière à pouvoir les comparer directement deux à deux (amont-aval Toulouse et bras supérieur-bras inférieur). Les principales caractéristiques des stations sont présentées dans le Tableau 1.

Les secteurs 1 et 4 présentent un écoulement lotique, dans un lit naturel bordé d'un rideau de végétation. Ils ont des caractéristiques morphodynamiques de pente $(>1,3 \%$ o), de température maximale du mois le plus chaud $\left(22\right.$ à $\left.26^{\circ} \mathrm{C}\right)$, de distance à la source $(140 \mathrm{~km}$ et $190 \mathrm{~km}$ ) et de largeur (environ $100 \mathrm{~m}$ ), qui les placent au niveau 'B8' de la typologie de Verneaux (1981). Cette portion de Garonne correspond à la zone à barbeau inférieur selon Huet (1949).

Les secteurs 2 et 3 , de longueur sensiblement identique $(4 \mathrm{~km})$, ont des écoulements lentiques par moyennes et basses eaux. Leur caractéristiques les placent au niveau typologique 'B9' (Verneaux 1981). Ils se départagent à l'amont par une chaussée qui surverse pour un débit entrant supérieur à $190 \mathrm{~m}^{3} \cdot \mathrm{s}^{-1}$. En dehors des situations de surverse, le bras inférieur n'est alimenté à son origine que par un faible débit réservé $\left(0.3 \mathrm{~m}^{3} \cdot \mathrm{s}^{-1}\right)$ et par les rejets industriels permanents (environ $\left.10 \mathrm{~m}^{3} \cdot \mathrm{s}^{-1}\right)$. Le bras inférieur fût longtemps abiotique (Gisclard 1984). La principale activité polluante concerne une usine de fertilisants azotés pour l'agriculture. Les actions de réduction des rejets à la source sont passées de $14800 \mathrm{~kg} \cdot \mathrm{j}^{-1}$ d'azote total en 1982 à $3655 \mathrm{~kg} . \mathrm{j}^{-1}$ en 1992 (données DRIRE Midi-Pyrénées).

Le bras supérieur, en continuité avec la Garonne à son extrémité amont, se termine à l'aval par une chaussée et une usine hydroélectrique équipée en passe à poissons. Le bras inférieur correspond au lit naturel consolidé alors que le bras supérieur est un canal plus profond, conçu pour les divers usages de l'eau. Les deux bras possèdent des portions de berges plus ou moins aménagées et suffisamment de diversité pour y établir des stations d'étude morphologiquement homologues.

Le débit moyen de la Garonne à Toulouse est de 191 $\mathrm{m}^{3} \cdot \mathrm{s}^{-1}$ (entre 1970 et 1993). Les étiages sont sévères en été (débit moyen mensuel de $33,87 \mathrm{~m}^{3} \cdot \mathrm{s}^{-1}$ en août 


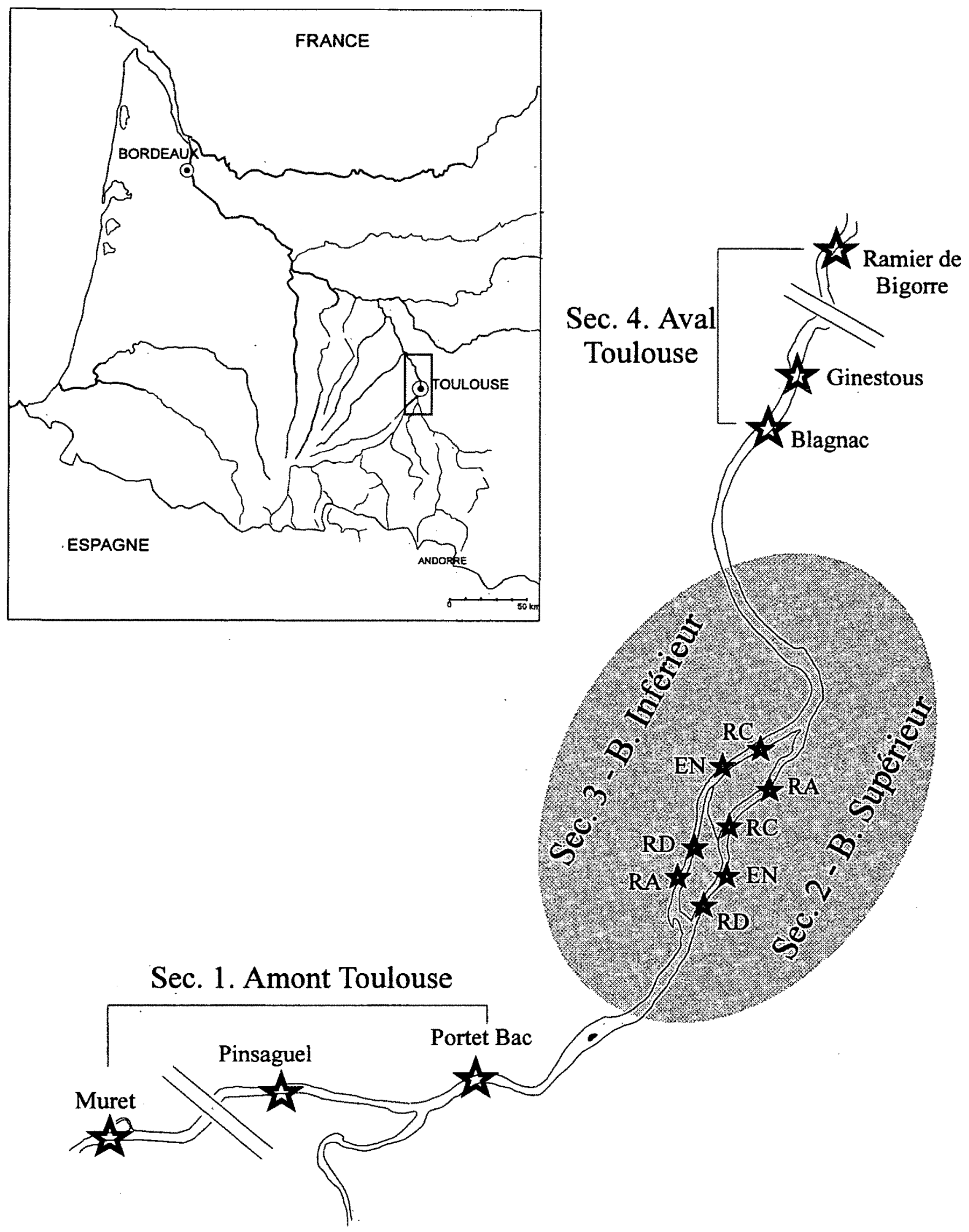

Fig. 1. Situation géographique des secteurs et stations d'études.

Fig. 1. Map of the sectors and sites studied. 
Tableau 1. Caractéristique des secteurs et stations d'étude.

Table 1. General description of sectors and sites studied.

\begin{tabular}{|c|c|c|c|}
\hline Secteurs (codes) & Ecoulement* & Stations & Caractéristiques \\
\hline $\begin{array}{c}1 \text { - Amont Toulouse } \\
\text { (Sec. 1) }\end{array}$ & lotique & $\begin{array}{l}\text { Portet-bac - PB } \\
\text { Pinsaguel - PG } \\
\text { Muret - MR }\end{array}$ & $\begin{array}{l}\text { Radier varié sur dalle }_{1}::_{1} \\
\text { Radier varié sur galets } \\
\text { Abris de type embâcles près de berge. }\end{array}$ \\
\hline $\begin{array}{c}2 \text { - Bras supérieur } \\
\text { (Sec. 2) }\end{array}$ & lentique & $\begin{array}{l}\text { Ripisylve dense - RD } \\
\text { Ripisylve moyenne - RM } \\
\text { Ripisylve absent - RA } \\
\text { Enrochement - EN }\end{array}$ & $\begin{array}{l}\text { Abris de type racines et embâcles } \\
\text { Abris de type embâcles } \\
\text { Abris de type végétation aquatique } \\
\text { Abris de type enrochement des berges }\end{array}$ \\
\hline $\begin{array}{c}\text { 3- Bras inférieur } \\
\text { (Sec. 3) }\end{array}$ & lentique & $\begin{array}{l}\text { Ripisylve dense - RD } \\
\text { Ripisylve moyenne - RM } \\
\text { Ripisylve absent - RA } \\
\text { Enrochement - EN }\end{array}$ & $\begin{array}{l}\text { Abris de type racines et embâcles } \\
\text { Abris de type embâcles } \\
\text { Abris de type végétation aquatique } \\
\text { Abris de type enrochement }\end{array}$ \\
\hline $\begin{array}{c}4 \text { - Aval Toulouse } \\
\text { (Sec. 4) }\end{array}$ & lotique & $\begin{array}{l}\text { Blagnac - BG } \\
\text { Ginestous - GT } \\
\text { Ramier de Bogorre - RB }\end{array}$ & $\begin{array}{l}\text { Radier varié sur dalle } \\
\text { Radier varié sur galets } \\
\text { Abris de type embâcles près de berges }\end{array}$ \\
\hline
\end{tabular}

*) Type d'écoulement par basses eaux

1989). La plus forte crue, pendant la période d'étude, a atteint $1010 \mathrm{~m}^{3} \cdot \mathrm{s}^{-1}$ (12 janvier 1995), ce qui est peu par rapport à des crues antérieures $\left(>\right.$ de $2000 \mathrm{~m}^{3} \cdot \mathrm{s}^{-1}$ en 1992).

\section{Matériel et méthodes}

Deux campagnes d'échantillonnages par pêche ont été effectués dans les 4 secteurs précités, la première en étiage hivernal 1994 (température $13.2^{\circ} \mathrm{C}$; débit $110 \mathrm{~m}^{3} \cdot \mathrm{s}^{-1}$ ), la seconde en étiage estival 1995 (température $25.1^{\circ} \mathrm{C}$; débit $81 \mathrm{~m}^{3} \cdot \mathrm{s}^{-1}$ ).

\subsection{Capture des poissons}

Les différences de profondeur et de vitesse de courant ont conduit à diversifier les méthodes d'échantillonnage. La pêche électrique a été utilisée aux zones les moins profondes alors que la pêche aux filets est plus adaptée aux zones calmes $>$ à 1,5 m (Daget 1976).

\subsubsection{Pêche électrique}

Compte-tenu de la configuration des secteurs 1 et 4 , la pêche électrique a été pratiquée selon la protocole de l'Echantillonnage Ponctuel d'Abondance (Nelva et al. 1979, Nelva 1985, Persat et al. 1981, 1985). 50 à 100 points ont été échantillonnés à chaque station. L'unité d'effort de pêche est constitué par un sondage ponctuel. A chacun de ces points des variables environnementales ont été relevées (cf. 3.2.).

\subsubsection{Pêche aux filets}

Pour les stations appartenant aux secteurs 2 et 3 , la pêche a été pratiquée par une série de 11 filets maillants de fond, de $10 \mathrm{~m}$ de long, de 1,5 à $2,5 \mathrm{~m}$ de hauteur et de vide de maille entre 10 et $60 \mathrm{~mm}$ ont été placés perpendiculairement à la berge. Le temps de pose a varié entre 20 minutes et 2 heures, selon les mailles utilisées. Ces pêches aux filets ont été répétées 2 fois à chaque secteur, station et saison. Les caractéristiques environnementales ont été relevées à l'emplacement de chaque filet posé (soit 88 relevés). Pour mieux évaluer les limites des échantillonnages par filets (EPF), quelques pêches électriques selon le protocole des échantillonnages ponctuels (EPA) ont été occasionnellement effectuées sur les bordures des secteurs 2 et 3 à Toulouse.

Les poissons capturés ont été identifiés, mesurés au millimètre près et pesés au gramme près avant d'être relâchés sur place.

\subsection{Variables environnementales}

Pour chaque point de pêche en milieu lotique (EPA) et chaque emplacement de filet en milieu lentique (EPF), des caractéristiques ponctuelles de l'habitat ont été notées selon une codification quantitative (Tableau 2) ou semi-quantitative (présence/absence) (Tableau 3). Il s'agit des vitesses de courant, de la profondeur, 
de la nature du substrat, de l'abondance d'abris. Ces relevés ont conduit à distinguer 3 types de macrohabitats (radier sur dalle [RD], radier sur galet [RG] et macrohabitat riche en abris $[\mathrm{AB}]$ ), pour les secteurs lotiques et 4 types de macrohabitats (ripisylve dense [RD], ripisylve clairsemée [RC], ripisylve absente [RA], et enrochement [EN]), pour les secteurs lentiques. La connaissance de l'environnement a été complétée de données analytiques communiquées par l'Agence de l'Eau Adour-Garonne (Tableau 4).

\subsection{Traitements des donnés}

\subsubsection{Structure du peuplement}

Dans les secteurs 1 et 4 , la constance C (taux d'occurrence) des espèces dans le secteur est le pourcentage de points EPA (toutes campagnes confondues) où l'espèce a été rencontrée. Dans les secteurs 2 et 3 (pêche aux filets), la constance est définie comme le pourcentage de mesures où l'espèce a été prise dans la série de filets (toute campagne et tout macrohabitat confondus).

La structure du peuplement est décrite par l'indice de diversité de Shannon $\left[\mathrm{I}_{\mathrm{Sh}}\right]$ (Daget 1976) et l'Equitabilité [E] (Alatalo 1981, Ramade 1981):

$$
\begin{aligned}
& \mathrm{I}_{\mathrm{Sh}}=-\sum_{\mathrm{i}=1}^{\mathrm{i}=\mathrm{s}} \frac{\mathrm{n}_{\mathrm{i}}}{\mathrm{N}} \times \log _{2} \frac{\mathrm{n}_{\mathrm{i}}}{\mathrm{N}} \quad \mathrm{E}=\mathrm{I}_{\mathrm{Sh}} / \log _{2} \mathrm{~S} \\
& \mathrm{~S}=\text { richesse spécifique } \\
& \mathrm{n}_{\mathrm{i}}=\text { effectif de l'espèce } \mathrm{i} \\
& \mathrm{N}=\text { effectif total du prélèvement. }
\end{aligned}
$$

La ressemblance entre deux peuplements (entre stations ou entre saisons) est évaluée par l'indice de similitude de Sørensen (Legendre \& Legendre 1979) :

So $=2 c /\left(S_{i}+S_{j}\right)$

$\mathrm{c}=$ nombre $\mathrm{d}$ 'espèces communes aux deux stations comparées.

$S_{i}$ et $S_{j}=$ nombre d'espèces des stations $i$ et $j$, respectivement.

Tableau 2. Principales caractéristiques des 3 stations du milieu lotique (secteurs 1 et 4).

Table 2. Main caracteristics observed at the 3 lotic stations (sectors 1 and 4).

\begin{tabular}{lccc}
\hline & Radier Dalle & Radier Galets & Abris \\
\hline Variables quantitatives* & & & \\
Distance à la berge $(\mathrm{m})$ & $1.9(0.7)$ & $2.4(0.8)$ & $2.7(0.7)$ \\
Profondeur $(\mathrm{m})$ & $59.7(25.4)$ & $40.7(18.6)$ & $46.1(21.7)$ \\
Vitesse de courant $(\mathrm{cm} / \mathrm{s})$ & $24.0(17.6)$ & $30.9(22.7)$ & $15.4(17.6)$ \\
Variables traitées sur la base de leur présence/absence** & & \\
Granulométrie & & & \\
Gros bloc & 1.1 & 1.1 & 0 \\
Petit bloc & 2.2 & 1.8 & 0.9 \\
Gros galet & 24.7 & 80.4 & 33.6 \\
Petit galet & 41.6 & 95.2 & 49.1 \\
Gravier & 10.1 & 22.6 & 17.3 \\
Sable & 5.6 & 3.6 & 4.5 \\
Vase & 1.1 & 2.4 & 16.4 \\
Dalle & 100 & 0 & 32.7 \\
Abris & & & \\
Absence & 62.9 & 55.4 & 0 \\
Abris constitués de blocs & 4.5 & 1.8 & 0 \\
Abris constitués de galets & 28.1 & 42.3 & 0 \\
Abris sous-berge & 0 & 0.6 & 14.5 \\
Abris consitués d'herbiers & 0 & 0 & 33.6 \\
Abris consitués de branches & 2.2 & 0 & 26.4 \\
Abris consitués de racines & 2.2 & 0 & 25.5 \\
\hline
\end{tabular}

*) Valeurs moyennes (ecart-type)

**) Pourcentage d'occurrence $(\mathrm{N}=370$ points de mesures) 
Tableau 3. Principales caractéristiques des 4 stations du milieu lentique (secteurs 2 et 3 ).

Table 3. Main caracteristics observed at the 4 lentic stations (sectors 2 and 3).

\begin{tabular}{|c|c|c|c|c|}
\hline \multirow{2}{*}{$\begin{array}{l}\text { Variables semi quantitatives (traitées } \\
\text { sur la base de leur présene/absence)* }\end{array}$} & \multicolumn{4}{|c|}{ Stations } \\
\hline & Ryp. Dense & Ryp. Clairsemée & Ryp. Absente & Enrochement \\
\hline \multicolumn{5}{|l|}{ Rive } \\
\hline 1. A pente faible & 22.5 & 52.3 & 52.3 & 40.9 \\
\hline 2. A pente forte & 77.3 & 47.7 & 47.7 & 59.1 \\
\hline \multicolumn{5}{|l|}{ Profondeur } \\
\hline 1. $<1 \mathrm{~m}$ & - & 12.5 & 11.3 & 20.5 \\
\hline 2.1 à $2 \mathrm{~m}$ & 30.6 & 53.4 & 51.1 & 39.8 \\
\hline 3. $>2 \mathrm{~m}$ & 69.4 & 34.1 & 37.6 & 39.8 \\
\hline \multicolumn{5}{|l|}{ Substrat } \\
\hline 1. Vase-sable & 25 & 59.1 & 29.5 & 21.6 \\
\hline 2. Molasse & 45.5 & 20.5 & 1.1 & 6.8 \\
\hline 3. Graviers-galets & - & - & 38.6 & 1.1 \\
\hline 4. Blocs & - & - & 1.1 & 53.4 \\
\hline 5. Vase-sable et molasse & 29.5 & 20.5 & - & - \\
\hline 6. Vase-sable et graviers-galets & - & - & 29.5 & - \\
\hline 7. Vase-sable et blocs & - & - & - & 17 \\
\hline \multicolumn{5}{|l|}{ Abri } \\
\hline 1. Absente & 27.3 & 26.1 & 45.5 & - \\
\hline 2. Racine-souche & 72.7 & 73.9 & 52.3 & - \\
\hline 3. Enrochement & - & - & 2.3 & 100 \\
\hline \multicolumn{5}{|l|}{ Végétation aquatique } \\
\hline 1. Absence & 97.7 & 85.2 & 36.4 & 75 \\
\hline 2. Présence & 2.3 & 14.8 & 63.6 & 25 \\
\hline \multicolumn{5}{|l|}{ Ombrage } \\
\hline 1. Absence & 26.1 & 23.9 & 79.5 & 76.1 \\
\hline 2. Présence & 73.9 & 76.1 & 20.5 & 23.9 \\
\hline \multicolumn{5}{|l|}{ Berge } \\
\hline 1. A pente faible & 21.6 & 64.8 & 77.3 & 14.8 \\
\hline 2. A pente forte & 78.4 & 35.2 & 22.7 & 85.2 \\
\hline \multicolumn{5}{|l|}{ Vitesse de courant } \\
\hline 1. Faible $(<5 \mathrm{~cm} / \mathrm{s})$ & 44.3 & 58 & 37.5 & 36.4 \\
\hline 2. Moyenne $(5$ à $10 \mathrm{~cm} / \mathrm{s})$ & 38.6 & 38.6 & 43.2 & 45.5 \\
\hline 3. Forte $(>10 \mathrm{~cm} / \mathrm{s})$ & 17 & 3.4 & 19.3 & 18.2 \\
\hline
\end{tabular}

* pourcentages d'occurence $(\mathrm{N}=352$ points de mesures)

Tableau 4. Physico-chimie de l'eau des 4 secteurs étudiés.

Table 4 . Water quality of the 4 sectors studied.

\begin{tabular}{lcccccccc}
\hline Paramètres & \multicolumn{2}{c}{ Secteur 1 } & \multicolumn{2}{c}{ Secteur 2 } & \multicolumn{2}{c}{ Secteur 3 } & \multicolumn{2}{c}{ Secteur 4 } \\
\cline { 2 - 9 } & Etée & Hiver & Eté & Hiver & Eté & Hiver & Eté & Hiver \\
\hline Température $\left({ }^{\circ} \mathrm{C}\right)$ & 20.25 & 10.05 & 19.9 & 10.85 & 25.4 & 16.85 & 22.55 & 11.02 \\
pH (unité pH) & 8.15 & 8.25 & 7.85 & 8.6 & 7.05 & 7.55 & 8.15 & 8.3 \\
Conductivité $(\mu \mathrm{S} / \mathrm{cm})$ & 205 & 235 & 194.5 & 225 & 409.5 & 500 & 209 & 275 \\
Oxygène dissous $(\mathrm{mg} / \mathrm{l})$ & 8.2 & 11.3 & 8.45 & 12.7 & 6.7 & 8 & 9.75 & 8.65 \\
NH4 $(\mathrm{mg} / \mathrm{l})$ & 0.125 & 0.09 & 0.15 & 0.07 & 1.385 & 1.75 & 0.335 & 0.83 \\
NO2 $(\mathrm{mg} / \mathrm{l})$ & 0.035 & 0.02 & 0.045 & 0.03 & 0.5 & 0.455 & 0.235 & 0.195 \\
NO3 $(\mathrm{mg} / \mathrm{l})$ & 1.75 & 2.6 & 2.4 & 3.25 & 10.15 & 13.4 & 4.65 & 6.5 \\
PO4 $(\mathrm{mg} / \mathrm{l})$ & 0.02 & 0.06 & 0.065 & 0.075 & 0.16 & 0.295 & 0.22 & 0.305 \\
\hline
\end{tabular}


La proportion numérique de poissons fourrage/poissons carnassier est quantifiée par le coefficient de Swingle (1950) :

$\mathrm{Cs}=\mathrm{F} / \mathrm{C}$

$\mathrm{F}=$ biomasse de poissons fourrage

$\mathrm{C}=$ biomasse de poissons carnassiers.

\subsubsection{Abondance}

Pour la pêche électrique, les captures sont exprimées en effectifs de poissons capturés par EPA (Nelva et al. 1979). Pour les pêches aux filets, les captures sont exprimées par unité d'effort de pêche (CPUE) définie comme le nombre d'individus capturés par $\mathrm{m}^{2}$ de filet en action pendant 1 heure. Il s'agit de la mesure la plus immédiate de l'abondance des captures (Laurec et al. 1983, Welcomme 1985, Changeux 1994).

La distribution des données ne satisfaisant pas aux critères de normalité, les différences d'abondance observées entre les stations et les saisons ont été testées selon les méthodes statistiques non paramétriques (Tests de Mann-Whitney et Kruskal-Wallis).

Des analyses multivariées ont été mises en oeuvre pour rechercher l'évolution spatio-temporelle de l'abondance des poissons en fonction des habitats physiques. La comparaison de l'abondance totale par relevé (somme marginale des lignes) à un sens pour nos données puisque, d'une part, l'effort d'échantillonnage est standardisé et d'autre part, pour chaque espèce, la variable mesurée (l'abondance) est la même. Cette comparaison des marges nous intéresse car la typologie des relevés doit être quantitative. Ceci nous conduit donc à éliminer les méthodes statistiques qui suppriment la variabilité des sommes marginales des lignes dont l'AFC (Poizat 1993).

Nous voulons décrire de quelle manière les relevés se distinguent de l'hypothèse nulle d'une répartition uniforme des espèces dans des relevés. Cette hypothèse nulle correspond à la référence d'une ACP centrée par colonne (par espèce) (Dolédec \& Chessel 1991, Poizat 1993). Les analyses multivariées sont réalisées à l'aide de la programmathèque ADE (Thioulouse et al. 1994, Thioulouse et al., sous-presse).

\section{Résultats}

\subsection{Composition du peuplement}

Par les deux méthodes confondues, 27 espèces ont été capturées dans l'ensemble des 4 secteurs (Tableau 5). Vingt espèces sont recensées en milieu lentique et 23 en milieu lotique. Huit espèces seulement sont communes aux 4 secteurs.

En milieu lotique (secteurs 1 et 4 ), le peuplement est dominé par le goujon, le barbeau et le chevaine, es- pèces caractéristiques de la Zone à Barbeau de Huet (1949), et marqué par la présence d'espèces typiques des milieux lotiques telles la truite commune, le vairon, le barbeau, la lamproie de planer et la loche franche. Mais des espèces plus spécifiques des milieux lentiques telles l'anguille, la gambusie et la bouvière sont également présentes (Fig. 2). Sur les 23 espèces capturées en milieu lotique, deux espèces sont constantes à plus de $30 \%$ (barbeau et goujon) et 4 espèces ont une constance comprise entre 10 et $30 \%$ (chevaine, toxostome, ablette, anguille). Les 17 autres espèces ont été capturées plus occasionnellement $(\mathrm{C}<$ $10 \%$ ) (Fig. 2).

En milieu lentique (secteurs 2 et 3), le peuplement recensé par pêches aux filets est dominé, en nombre, par plus de $20 \%$ de gardons et, à un degré moindre, par la brème, l'ablette, le sandre, la vandoise, le rotengle et la grémille (Fig. 2). Les contrôles effectués occasionnellement par EPA près des berges indiquent aussi la présence d'anguilles et de bouvières. D'une manière générale, les espèces sont plus constantes dans les secteurs 2 et 3 qu'en milieu lotique. Sur les 20 espèces de milieu lentique, 9 sont constantes à plus de $50 \%$ dans le bras inférieur (chevaine, brème, toxostome, gardon, carassin, ablette, sandre, perche commune, perche soleil), 9 autres sont accessoires et 2 sont occasionnelles (Fig. 2). A noter la présence du gardon d'Albanie ( $\mathrm{Pa}$ chychilon pictus), une espèce non autochtone, originaire d'Albanie. La constance des espèces est moindre dans le bras supérieur où seulement 5 espèces ont été recensées dans $50 \%$ des pêches (chevaine, gardon, brème, ablette, perche).

Les variations saisonnières sont évidentes pour les secteurs 1 et 2 , avec une richesse spécifique (Tableau 6) moindre en hiver ( 3 espèces) par rapport à l'été bien que l'échantillonnage ait été réalisé à débit similaire. Pour les secteurs 3 et 4 , le nombre des espèces capturées est identique aux deux saisons (15 espèces).

La diversité spécifique, traduite par l'indice de Shannon, confirme que les peuplements sont mieux équilibrés en secteurs lotiques : $\mathrm{I}_{\mathrm{Sh}}$ moyen $=3,19$ qu'en secteurs lentiques : $\mathrm{I}_{\mathrm{Sh}}$ moyen $=2,90$ (Tableau 6 ).

Le coefficient de Swingle en milieu lotique varie selon la saison ; il est en moyenne de 2,8 en été, et de 9,6 en hiver. En milieu lentique ce coefficient varie selon les secteurs, dans une gamme comprise entre 3,0 pour le secteur 3 et 13,5 pour le secteur 2 . Pour le secteur 3 , ce coefficient est sensiblement constant d'une saison à l'autre.

Les fortes similitudes qualitatives entre d'une part, les secteurs 1 et 4 en milieu lotique et d'autre part, les secteurs 2 et 3 en milieu lentique (So $>0,70$ ) révèlent 
Tableau 5. Liste des espèces et leur caractéristiques écologique et trophique aux 4 secteurs étudiés.

Table 5. List of species and their ecologic and trophic caracteristics in the 4 sectors studied.

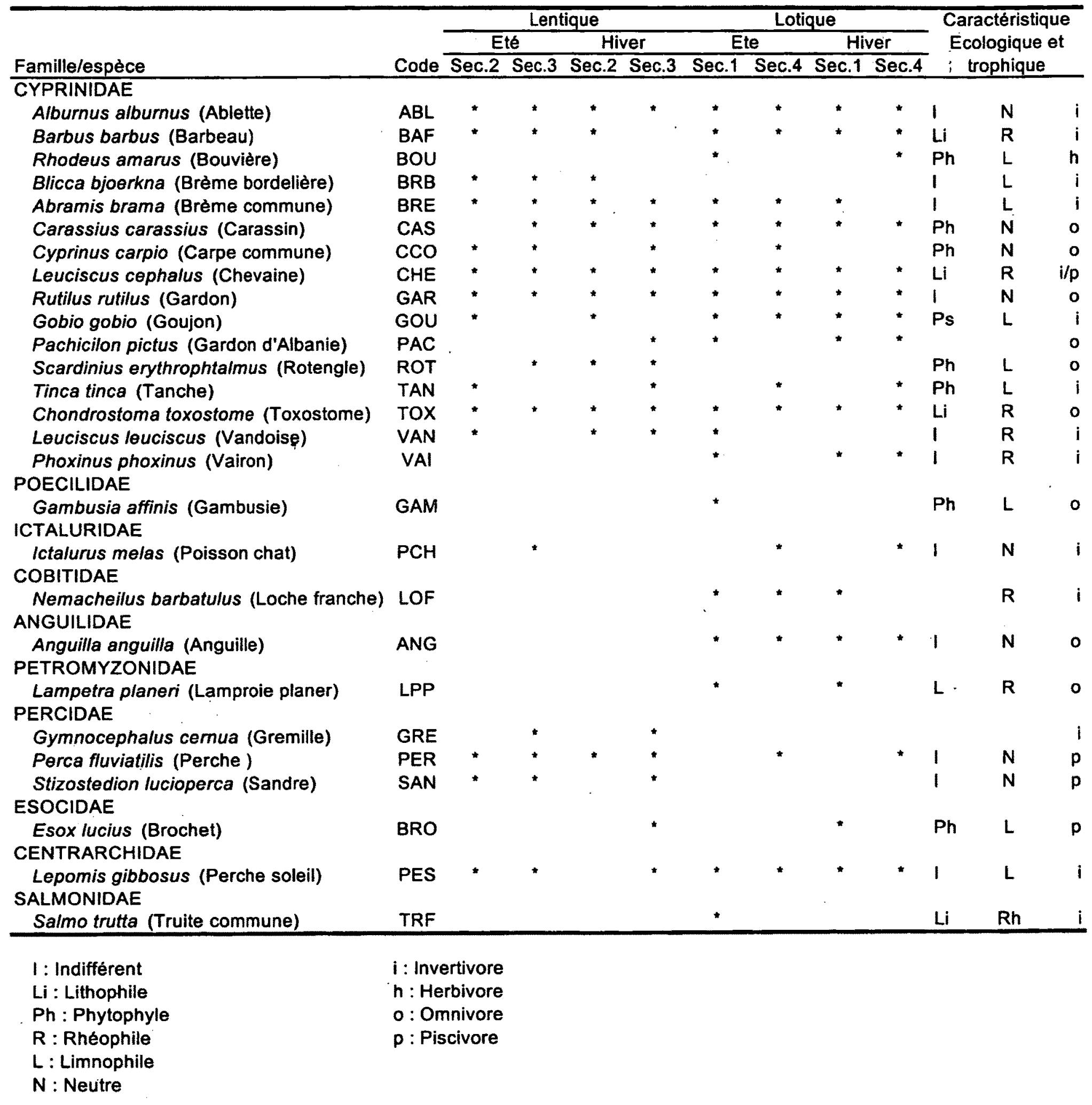



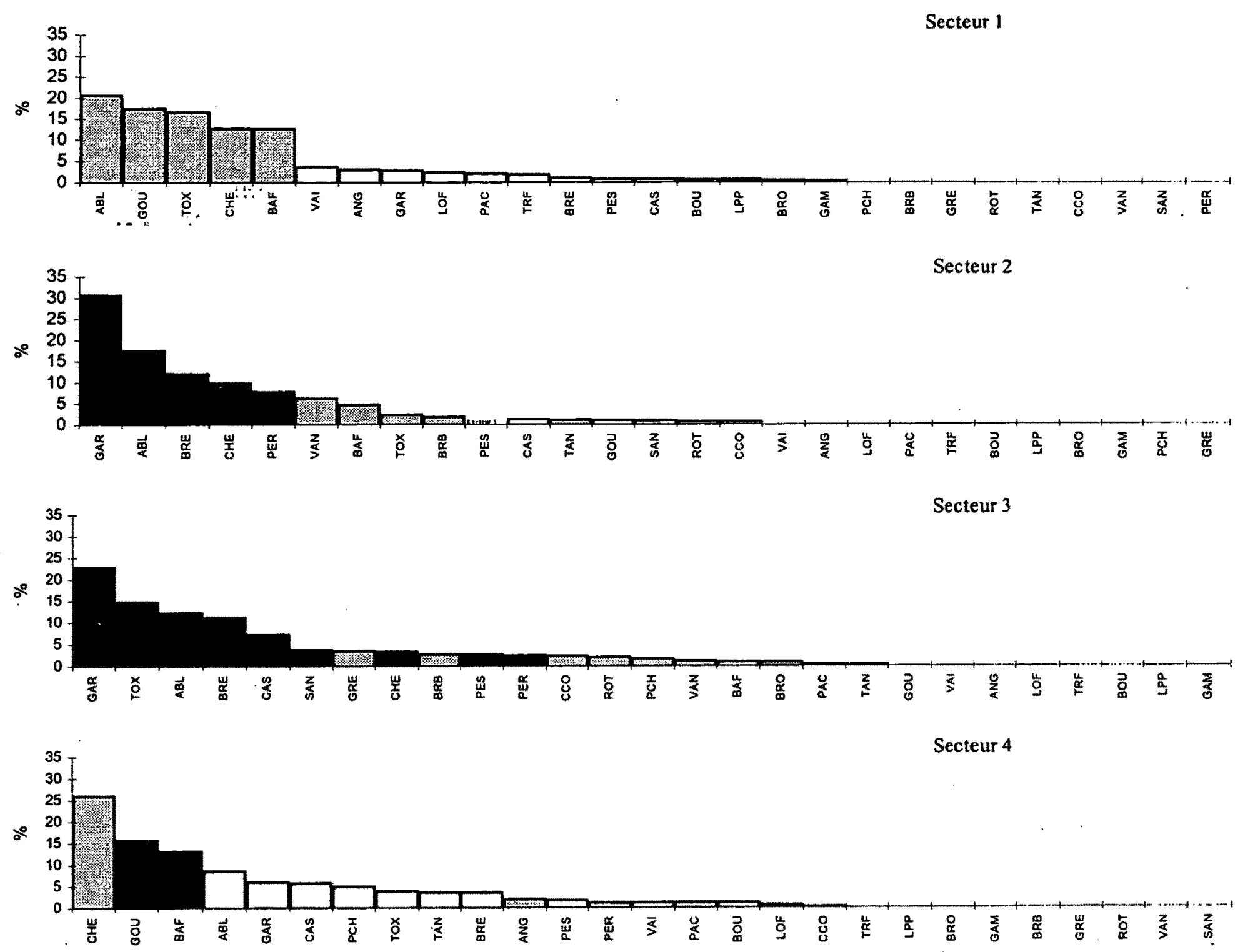

Fig. 2. Fréquence des espèces (\%) aux 4 secteurs étudiés. Les barres de couleur noire désignent les espèces constantes, barrẹs grise les espèces accessoires, barres blanche les espèces accidentelles.

Fig. 2. Species frequency (\%) at the 4 sectors studied. Black bars showed constant species, grey bars secondary species and white bars rare species.

Tableau 6. Richesse spécifique (RS), Indice Shannon $\left(\mathrm{I}_{\mathrm{SH}}\right)$, Equitabilité (E) et coefficient de Swingle (CS) des 4 secteurs d'étude.

Table 6. Specific richness (RS), Shannon index $\left(\mathrm{I}_{\mathrm{SH}}\right)$, Equitability $(\mathrm{E})$ and coefficient of Swingle (CS) of the 4 sectors studied.

\begin{tabular}{|c|c|c|c|c|c|c|c|c|}
\hline & \multicolumn{4}{|c|}{ Lotique } & \multicolumn{4}{|c|}{ Lentique } \\
\hline & \multicolumn{2}{|c|}{ Eté } & \multicolumn{2}{|c|}{ Hiver } & \multicolumn{2}{|c|}{ Eté } & \multicolumn{2}{|c|}{ Hiver } \\
\hline & Sec. 1 & Sec. 4 & Sec. 1 & Sec. 4 & Sec. 2 & Sec. 3 & Sec. 2 & Sec. 3 \\
\hline RS & 17 & 14 & 15 & 15 & 14 & 15 & 11 & 16 \\
\hline ISH & 3.46 & 3.39 & 2.93 & 3.00 & 2.29 & 2.76 & 2.49 & 2.85 \\
\hline $\mathrm{E}$ & 0.85 & 0.89 & 0.75 & 0.77 & 0.60 & 0.71 & 0.72 & 0.71 \\
\hline CS & 2.7 & 2.91 & 13.07 & 6.1 & 14.76 & 3.97 & 12.14 & 2.09 \\
\hline
\end{tabular}


Tableau 7. Indice de similitude de Sørensen des 4 secteurs, aux 2 saisons.

Table 7. The similarity index of Sørensen at the 4 sectors studied of the 2 seasons.

\begin{tabular}{lcccccccc}
\hline & $\begin{array}{c}\text { Hiver- } \\
\text { Sect.1 }\end{array}$ & $\begin{array}{c}\text { Hiver- } \\
\text { Sect.2 }\end{array}$ & $\begin{array}{c}\text { Hiver- } \\
\text { Sect.3 }\end{array}$ & $\begin{array}{c}\text { Hiver- } \\
\text { Sect.4 }\end{array}$ & $\begin{array}{c}\text { Eté- } \\
\text { Sect.1 }\end{array}$ & $\begin{array}{c}\text { Eté- } \\
\text { Sect.2 }\end{array}$ & $\begin{array}{c}\text { Eté- } \\
\text { Sect.3 }\end{array}$ & $\begin{array}{c}\text { Eté- } \\
\text { Sect.4 }\end{array}$ \\
\hline Hiver-Sec.1 & 1 & & & & & & & \\
Hiver-Sec.2 & 0.54 & 1 & & & & & & \\
Hiver-Sec.3 & 0.58 & 0.67 & 1 & & & & & \\
Hiver-Sec.4 & $\mathbf{0 . 7 3}$ & 0.54 & 0.58 & 1 & & & & \\
Eté-Sec.1 & $\mathbf{0 . 8 8}$ & 0.50 & 0.48 & $\mathbf{0 . 7 5}$ & 1 & & & \\
Eté-Sec.2 & 0.55 & $\mathbf{0 . 7 2}$ & $\mathbf{0 . 7 3}$ & 0.62 & 0.52 & 1 & & \\
Eté-Sec.3 & 0.53 & $\mathbf{0 . 7 7}$ & $\mathbf{0 . 7 7}$ & 0.60 & 0.50 & $\mathbf{0 . 7 6}$ & 1 & \\
Eté-Sec.4 & $\mathbf{0 . 7 6}$ & 0.64 & 0.60 & 0.83 & $\mathbf{0 . 7 1}$ & 0.70 & 0.69 & 1 \\
\hline
\end{tabular}

les affinités spécifiques du peuplement piscicole pour un type de milieu (Tableau 7). De plus, le peuplement des milieux lotiques est différent de celui des milieux lentiques (So $<0,50$ ).

\subsection{Abondance relative}

L'abondance de poissons (toutes espèces confondues) aux 4 secteurs étudiés et pour chaque saison est représentée dans la figure 3 . Pour les secteurs lotiques (1 et 4), aucune différence significative n'apparaît ni entre l'hiver (9.80 ind./EPA en moyenne) et l'été (4,76 ind./EPA) $(\mathrm{P}=0,078)$, ni entre le secteur amont et le secteur aval Toulouse $(P=0,2)$.

Pour les secteurs lentiques ( 2 et 3 ), des différences significatives existent entre les 2 bras de Garonne à Toulouse ( $P=0.002)$, mais non entre l'hiver et l'été $(P=0,4)$. Dans le bras inférieur (secteur 3$), 9.6$ ind. $/ \mathrm{m}^{2}$ de filet/heure (CPUE) ont été pêchés (moyenne des 2 saisons) contre 4.5 ind.$/ \mathrm{m}^{2}$ de filet/heure dans le bras supérieur (secteur 2).

Les résultats de l'ACP effectuée pour chaque type de milieu (lotique et lentique) sont présentés dans les figures 4 et 5 .

Pour les milieux lotiques (Fig. 4), l'essentiel de l'information est concentré sur les 2 premiers axes qui rendent compte de $63 \%$ de l'inertie totale ( $40 \% \mathrm{~F} 1$; $23 \%$ F2). Le premier axe est construit essentiellement par l'opposition entre le poisson-chat (présent secteur 4) et l'ablette, le toxostome et la loche franche (surtout présents secteur 1) (Figs. 4A, 4B). L'axe F1 montre donc une discontinuité des peuplements amont-aval de la ville de Toulouse.

Le deuxième axe est construit essentiellement par le barbeau, le chevaine, l'anguille et le poisson-chat. Au niveau des stations (Fig. 4D), on constate une opposition entre les macrohabitats contenant des abris (stations $\mathrm{MR}$ et RB) et les macrohabitats à radier sur dalle (stations PB et BG). Les macrohabitats à radier sur galets (stations PG et GT) se situent en position intermédiaire, à l'intersection des 2 premiers axes, ce qui signifie une absence de préférence particulière. Le deuxième axe traduit donc la différence de qualité des habitats, selon leur capacité à abriter les poissons.

En considérant chaque espèce, les différences entre les saisons (Fig. 4C) sont moins nettes que les effets «secteurs» et «stations».

Pour les milieux lentiques (Fig. 5), l'essentiel de l'information est concentré sur les 2 premiers axes qui rendent compte de $57 \%$ de l'inertie totale ( $37 \%$ F1, $20 \%$ pour F2). Le peuplement est dominé par le toxostome, le gardon, le carassin et l'ablette (Fig. 5A). L'abondance de ces 4 espèces change selon les saisons, les secteurs et les stations. On les retrouve principalement dans le bras inférieur (secteur 3) (Fig. 5C, axe F1 partie négative). En hiver, le toxostome et le carassin dominent dans les stations RC et RD (Fig. 5B et 5D, axe F2 partie positive). En été, le gardon et l'ablette dominent dans les stations RA et EN (Fig. 5B et 5D axe F2 partie 

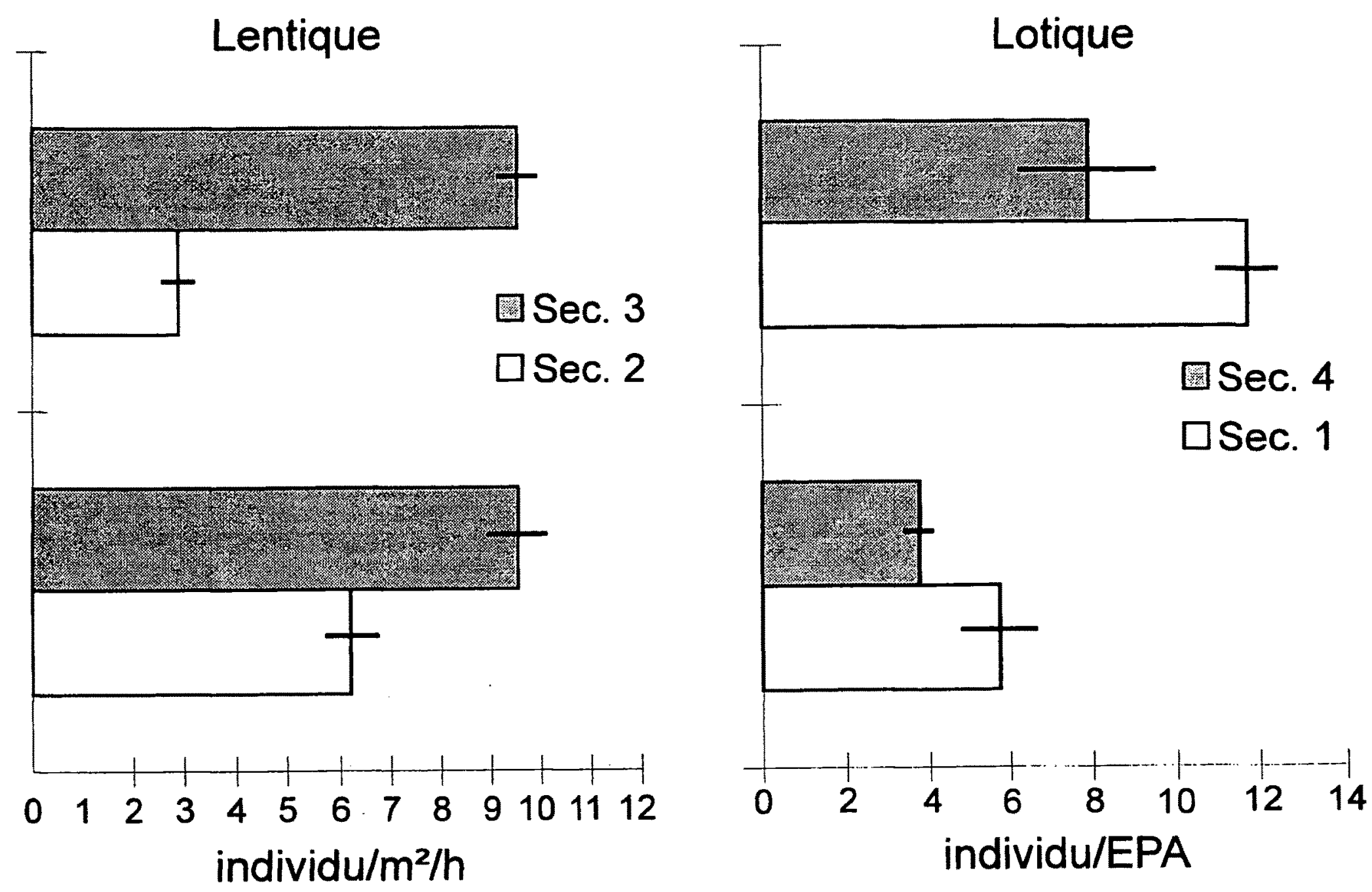

Fig. 3. Abondance de poissons (moyenne $( \pm \mathrm{SE}$ ) dans le 4 secteurs étudiés (lotique et lentique) pour les 2 saisons (toutes espèces confondues).

Fig. 3. Fish abundance (mean ( \pm SE) at the 4 sectors studied (lotic and lentic) for the 2 saisons.

négative). L'axe $\mathrm{F} 1$ oppose donc le bras inférieur au bras supérieur (effet secteur) et l'axe F2 combine un effet des macrohabitats et un effet des saisons. L'hiver se superpose aux stations de macrohabitats RD et RM et l'été aux stations de macrohabitats RA et EN.

\section{Discussion}

\subsection{Validité des techniques et limites de l'interpré- tation}

Les caractéristiques physiques du milieu telles la profondeur, la conductivité et la largeur de la rivière agissent sur l'efficacité de la pêche électrique (Vibert 1968, Cross 1976). Les profondeurs d'eau de plus de $1,5 \mathrm{~m}$ dans les milieux lentiques déterminent le choix de la pêche aux filets (Bengen et al. 1992, Chappaz 1986, Degiorgi 1993). Les captures obtenues par EPA et par EPF aux deux saisons (hiver-été), avec 4735 poissons, toutes espèces et classes de taille confon- dues, constituent une base de données substantielle pour aborder la biodiversité et les premières tendances sur les relations poissons-habitats au niveau d'un tronçon de rivière fortement anthropisé. Cependant, dans l'absolu, l'usage de méthodes différentes d'échantillonnage apporte quelques biais dans les abondances observées : les très petits poissons $(<50 \mathrm{~mm})$ et l'anguille ont tendance à échapper aux EPF et quelques grands poissons installés dans les points profonds sont peu accessibles à l'EPA. En effet, la mobilité des poissons et l'efficacité des filets varient selon de multiples facteurs notamment la température qui agit directement sur l'intensité métabolique et donc sur les besoins nutritionnels. Ces biais sont à relativiser sachant que ce sont les espèces les moins probables (petites espèces lotiques en zones lentiques explorées aux filets) qui peuvent échapper à l'échantillonnage. La comparaison des deux milieux lentiques étudiés par EPF et celle des milieux lotiques échantillonés par EPA reste 


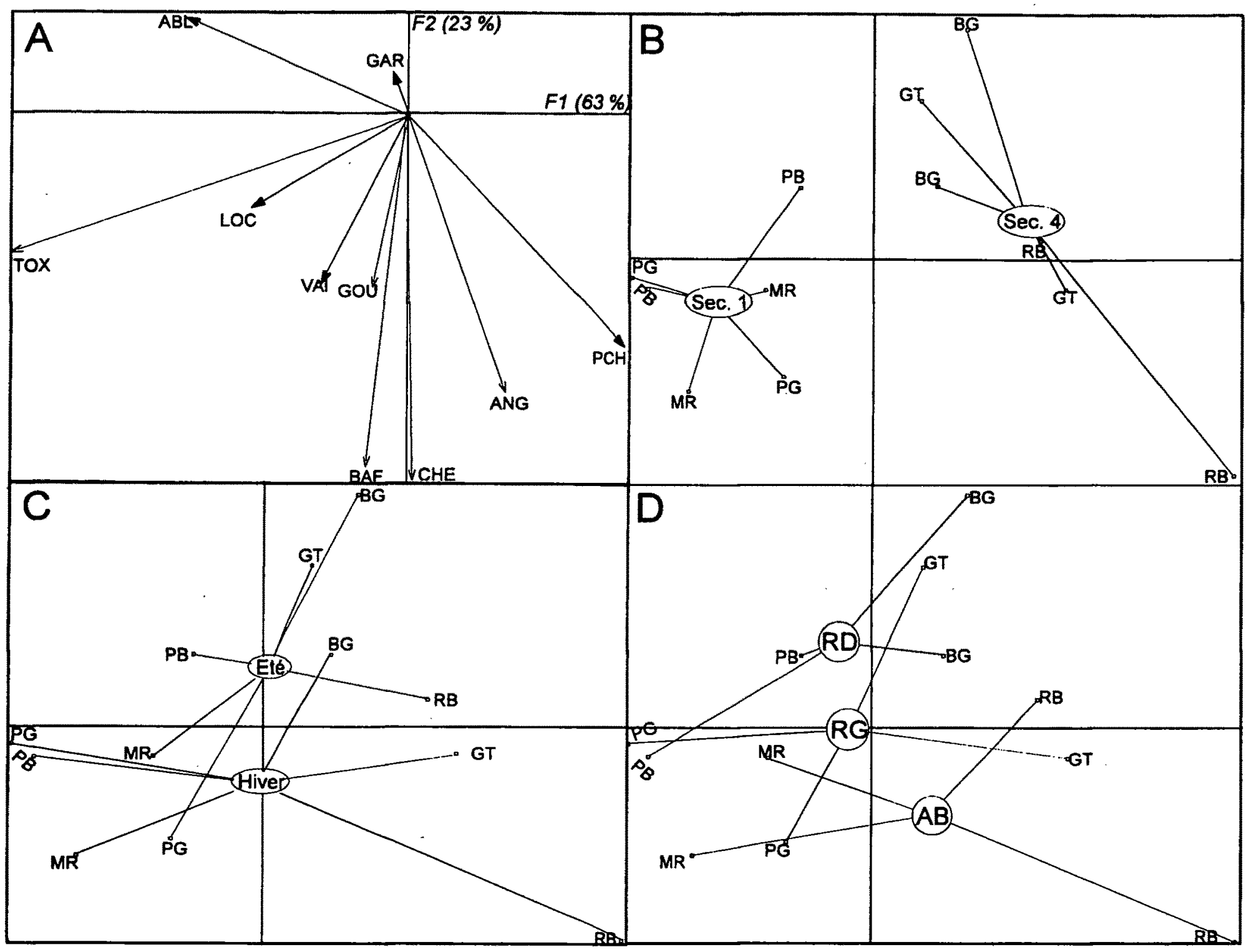

Fig. 4. Analyse en Composantes Principales (ACP) des abondances de poissons dans les secteurs lotiques. A. Représentation du cercle de corrélation (F1 x F2) des 23 espèces (abréviations voir Tableau 5). Représentation des individus regroupés par saisons (B), par secteurs (C) et par stations (D) (codes stations, voir Tableau 1).

Fig. 4. Principal Component Analysis (PCA) of fishe abundances at the lotic sectors. A. Representation of the correlation circle (F1 $x$ F2) of the 23 species (abreviations in Table 5). Representation of the individuals grouped by seasons. (B), by sectors (C) and by sites (D) (sites code, see Table 1).

valable, en connaissance des atouts et faiblesses de chaque méthode et pour des stations morphologiquement similaires.

\subsection{Diversité spécifique}

Les deux méthodes d'échantillonnage confondues (EPA et EPF) ont recensé 27 espèces, soit une diversité supérieure à celle (17 espèces) établie en 1985 par Lim et al. (1985) et comparable à celle observée en 1992 par Bengen (1992) (24 espèces recensées sur une étude de 2 ans , $50 \mathrm{~km}$ aval de Toulouse). La principale espèce manquante par rapport à cette dernière réfé- rence est le blackbass, poisson qui affectionne les milieux très calmes. En revanche, 4 espèces nouvelles le vairon, la loche franche, la truite commune et le gardon d'Albanie sont apparues. Cette dernière espèce a été introduite accidentellement avec des lots de goujons d'alevinage importés (Tertre, comm. pers.), et semble trouver ici des conditions de milieu très favorables à sa reproduction et à son développement. Des cas similaires d'introduction accidentelle de ce poisson ont été signalés en Grèce (Stephanidis 1974) et en Italie dans la rivière Serchio (Delmastro \& Balma 1990). 


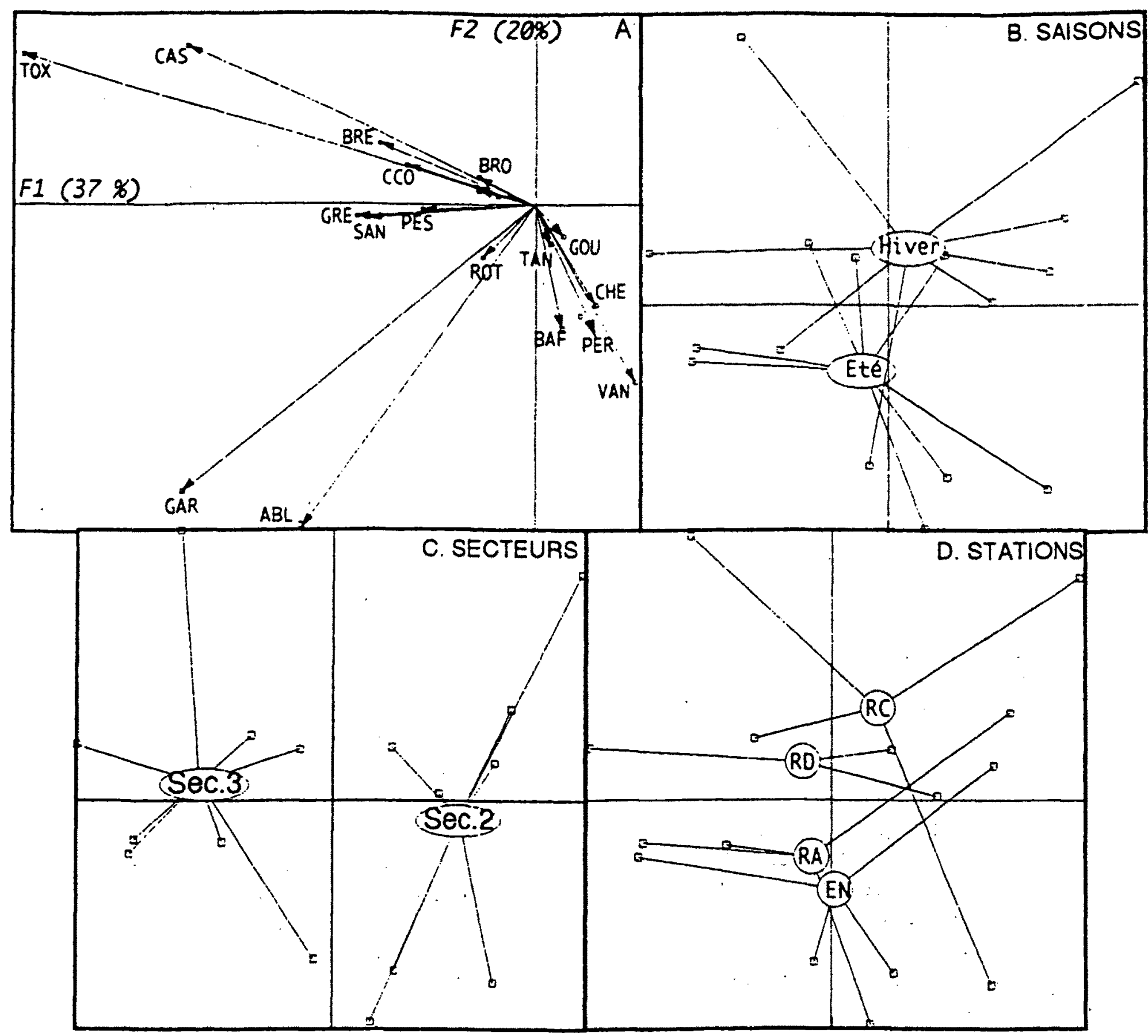

Fig. 5. Analyse en Composantes Principales (ACP) des abondances de poissons dans les secteurs lentiques. A. Représentation du cercle de corrélation (F1 x F2) des 20 espèces (abréviations voir Tableau 5). Représentation des individus regroupés par saisons (B), par secteurs (C) et par stations (D) (codes stations, voir Tableau 1).

Fig. 5. Principal Component Analysis (PCA) of fish abundances at the lentic sectors. A. Representation of the correlation circle (F1 X F2) of the 20 species (abreviations in Table 5), Representation of the individuals grouped by seasons (B), by sectors (C) and by sites (D) (site codes, see Table 1).

\subsubsection{Espèces dominantes}

Les secteurs 1 et 4 se situent au niveau «B8» de la typologie de Verneaux (1981) et correspondent à la «Zone à Barbeau» de Huet (1949). La différence entre les secteurs 1 et 4 porte sur la qualité de l'eau (Tableau 4), avec une température moyenne légèrement plus élevée (gradient climatique et effet de l'agglomération) et une pollution résiduelle au niveau du secteur aval.
Globalement, le peuplement de poissons comporte un certain nombre d'espèces communes aux deux secteurs lotiques, avec une dominance du barbeau, du chevaine et du goujon.

Les espèces constantes du secteur 1 (amont Toulouse) sont le barbeau, le chevaine, le toxostome, l'ablette et le goujon; celles du secteur 4 (aval Toulouse) sont le barbeau, le chevaine, le goujon et l'anguille (Fig. 2). 
L'anguille en aval et le toxostome et l'ablette en amont traduisent une évolution sensible des espèces dominantes. Ces dernières appartiennent, à l'exception de l'anguille, à la famille des Cyprinidae fréquentant les milieux courants. Selon Philippart (1977), elles se répartissent de manière très hétérogène selon les habitats et les densités du peuplement sont corrélées négativement avec la profondeur et la section mouillée de la rivière. Les indices de diversité de ces 2 secteurs sont relativement élevés, ce qui traduit l'absence de dominance importante d'une ou de plusieurs espèces. Dans ces 2 secteurs, l'équitabilité $\geq 0,75$ caractérise un peuplement équilibré (Daget 1976).

Le coefficient de Swingle apparait plus important en hiver qu'en été, ce qui peut tenir au fait que les carnassiers s'abritant en hiver en milieux profonds sont moins accessibles à la pêche électrique (Belaud et al. 1989, Bouchard 1996).

Les secteurs 2 et 3 présentent aussi une similitude morphodynamique. Bien qu'intercalés entre les secteurs lotiques 1 et 4 , ces deux secteurs présentent des caractéristiques morphodynamiques assimilables à des milieux de type «retenue» (pente faible, forte largeur). Le niveau typologique de ce segment de Garonne correspond au niveau «B9» selon Verneaux (1981) et à la «Zone à Brème» selon Huet (1949). La différence majeure entre les secteurs 2 et 3 porte sur la qualité des eaux. Le bras inférieur est soumis à une pollution ammoniacale en période de faibles eaux (débit inférieur à $190 \mathrm{~m}^{3} \cdot \mathrm{s}^{-1}$, soit $145 \mathrm{j} \cdot \mathrm{an}^{-1}$ ) de 4 à $6 \mathrm{mg} \cdot \mathrm{l}^{-1}$ (Lim et al. 1994). Dans ces mêmes conditions, les rejets thermiques accroissent la température de l'ordre de $5^{\circ} \mathrm{C}$ en été et $7^{\circ} \mathrm{C}$ en hiver dans le bras inférieur.

Le peuplement de poissons de ces 2 secteurs lentiques comprend des espèces communes avec dominance de gardón, de brème et d'ablette.

Les espèces les plus constantes du secteur 2 sont le chevaine, la brème, le gardon, l'ablette et la perche ; celles du secteur 3 sont le chevaine, la brème, le toxostome, le gardon, le carassin, l'ablette, le sandre, la perche commune, la perche soleil, soit 4 espèces de plus qu'au secteur 2 . Au vu des présents résultats, parmi les 9 espèces constantes du secteur 3 , la brème, le gardon et la perche sont plutôt représentatifs des zones calmes (Belaud et al. 1989). Lelek \& Köhler (1989) constatent que la majorité de ces espèces n'ont pas d'exigence particulière, ni vis-à-vis de la qualité d'eau, ni vis-à-vis des structures des habitats. Le gardon est un omnivore à capacité d'adaptation très élevée, qui peut survivre en milieu très limité en nourriture (Braband 1985), en exploitant toute ressource disponible (Michel \& Oberdorff 1995). Sa prolifération est pos- sible en milieux perturbés (Oberdorff \& Hughes 1992).

Les indices de diversité des secteurs 2 et 3 sont moins élevés que ceux des secteurs lotiques, ce qui signifie un moindre équilibre, avec dominance de quelques espèces (gardon, brème, ablette).

Les valeurs du coefficient de Swingle varient peu d'une saison à l'autre. En revanche, les valeurs 4 fois plus élevées dans le secteur 2 (Tableau 6) s'expliquent d'une part, par un prélèvement par les pêcheurs de carnassiers opérant au secteur 2 (et non au secteur 3 où la pêche est interdite) et d'autre part, par l'abondance particulière de poissons dans le bras inférieur qui sera discutée plus loin. La similitude morphodynamique des deux secteurs exclut un simple effet de qualité de l'habitat physique.

A ce stade de l'analyse, il apparaît que, bien que morphologiquement en Zone à Barbeau, le tronçon étudié présente une différence amont/aval visible dès le niveau des espèces principales, plus thermophiles et limnophiles en aval qu'en amont. Dans l'agglomération de Toulouse, les deux bras de la Garonne se singularisent par un écoulement lentique et des biocénoses différentes de celles de la partie libre du fleuve. Les différences de composition des espèces principales entre bras supérieur et bras inférieur sont le résultat des multiples actions humaines, dont le réchauffement qui attire des espèces thermophiles et.indifférentes à la pollution.

Cette approche, basée sur les espèces abondantes, montre déjà une singularité au niveau de l'agglomération de Toulouse, avec un tronçon lentique de typologie supérieure au segment originel. L'impact des activités humaines doit être affiné sur la base d'indices plus précis que la simple liste des espèces principales.

\subsubsection{Espèces occasionnelles}

Les espèces attendues dans la zone géographique étudiée mais plus occasionnelles dans les échantillonnages sont a priori des indicateurs précieux pour identifier les effets des activités humaines (les espèces principales sont présumées mieux adaptées). Les espèces occasionnelles en amont de Toulouse sont la vandoise, la gambusie, la lamproie de planer, le brochet et la truite commune ; les espèces occasionnelles en aval de Toulouse sont : la carpe commune, la tanche, le gardon d'Albanie et la perche commune. Ces nettes différences spécifiques paraissent importantes vu la similitude des milieux physiques (type 'B8', Verneaux 1981). Pour connaître s'il s'agit d'une évolution progressive sur le tronçon de $50 \mathrm{~km}$ ou si le changement s'opère brutalement dans la traversée de la ville, on s'appuiera sur la comparaison des deux bras 
de Garonne à Toulouse où les effets du réchauffement et de la pollution doivent se traduire par une discontinuité de la composition spécifique.

Au niveau de la ville, l'espèce occasionnellement capturée en été et en hiver dans le bras supérieur, non échantillonnée dans le bras inférieur et donc apparemment polluosensible, est le goujon. Les espèces exclusivement rencontrées dans le bras inférieur et donc a priori polluorésistantes et/ou thermophiles sont principalement le carassin, le poisson chat et la grémille. Le bras supérieur héberge à la fois des espèces occasionnelles rhéophiles, en continuité avec les secteurs amont et aval, et des espèces d'eau calme qui exploitent les milieux lentiques créés par la retenue. Les espèces qui sont installées dans le bras inférieur sont limnophiles et dotées d'une large gamme de tolérances écophysiologiques et écotoxicologiques. Une nette discontinuité typologique s'opère donc au niveau de la ville, en particulier au bras inférieur, avec un passage à écoulement lentique et un brusque changement de qualité de l'eau.

\subsection{Abondance}

En milieu lotique, on observe des variations d'abondance saisonnière entre l'étiage estival et l'étiage hivernal. L'abondance est de l'ordre de 10 ind./EPA en été et de l'ordre de 5 ind./EPA en hiver. Bengen et al. (1992) avaient pêché 3 bras morts de la Garonne et capturé en moyenne 11,7 ind./EPA, chiffre plus élevé que dans la présente étude mais obtenu sur des sites lentiques particulièrement riches en abris, très peuplés en alevins et en juvéniles.

Les densités des secteurs lentiques $\left(4.5 \mathrm{ind} . / \mathrm{m}^{2} \mathrm{de} \mathrm{fi-}\right.$ let $/ \mathrm{h}$, secteur 2 et $9.6 \mathrm{ind} . / \mathrm{m}^{2}$ de filet $/ \mathrm{h}$, secteur 3 ) peuvent être considérées comme élevées, en particulier dans le secteur 3 qui fut abiotique jusqu'en 1980 (Gisclard 1984). La qualité de ce secteur, après la réduction des pollutions à leur source ( $\mathrm{Lim}$ et al. 1994), s'est améliorée par le débit permanent de la Garonne dans le bras inférieur (de l'ordre de $0.3 \mathrm{~m}^{3} . \mathrm{s}^{-1}$ ), permettant sa recolonisation. D'un côté, les taux actuels de pollution restent potentiellement dangereux pour certaines espèces à certains stades, d'autant plus que la toxicité de l'ammoniaque est renforcée par des températures élevées (Emerson et al. 1975). D'un autre côté, il a été rapporté des cas de pollution subléthale où la biodiversité est plus élevée qu'en absence de pollution (Connell 1978). De même, Steedman (1988) constate des abondances plus élevées dans les rivières enrichies en matière organique ou réchauffées. La situation actuelle de la recolonisation est celle d'une forte abondance assurée par quelques espèces.
Le secteur $3\left(9,6\right.$ ind. $/ \mathrm{m}^{2}$ de filet/heure) paraît deux fois plus peuplé que le secteur $2\left(4,5 \mathrm{ind} / \mathrm{m}^{2}\right.$ de filet /heure). Les variations saisonnières montrent aussi plus de stabilité de l'abondance du peuplement pour le secteur 3. Ces valeurs élevées et stables du secteur 3 désignent une action favorable du facteur thermique et relèguent au second plan l'effet toxique de l'ammoniaque $\left(1,6 \mathrm{mg} . \mathrm{l}^{-1}\right.$ en moyenne annuelle ; 4 à $6 \mathrm{mg} . \mathrm{l}^{-1}$ par faibles débits). Ces tendances sur l'abondance moyenne des poissons sont susceptibles d'être biaisées par une probable plus grande mobilité des poissons dans le bras inférieur (effet de température et/ou de pollution). Une plus longue série de mesures, en particulier aux saisons où les températures sont équivalentes dans les deux bras, s'avère nécessaire pour conclure définitivement.

\subsection{Occupation de l'habitat au sein des stations}

Pour les espèces dominantes de chaque secteur, les résultats permettent de s'intéresser aux relations poisson/habitat physique au cours des saisons et d'observer d'éventuelles anomalies qui pourraient être en relation avec les caractéristiques de chaque secteur.

Entre l'amont et l'aval de Toulouse, la répartition du toxostome et du goujon diffère, notamment en hiver (toxostomes rassemblés dans les radiers sur dalles en aval; goujons rassemblés sur les radiers sur galets en aval), tandis que les autres espèces ont une répartition ubiquiste en toute saison. Les préférences de la majorité des Cyprinidae, Anguillidae et Ictaluridae vont aux stations riches en abris.

Au niveau de Toulouse, le gardon, le chevaine, la brème et l'ablette se répartissent de manière équilibrée entre les 4 types de macrohabitat définis par les variables environnementales relevées. Le toxostome, le gardon, le carassin et l'ablette, Cyprinidae d'eau calme, sont présents dans tous les habitats disponibles. Le toxostome et le carassin préfèrent les stations à ripisylve dense (RD) et moyenne (RC) en hiver. Par contre, le gardon et l'ablette préfèrent les stations à ripisylve absente. (RA), avec plantes aquatiques et enrochements (EN) en été. Cette répartition a également été observée dans le Rhône (Copp 1992).

Le toxostome effectue un réel choix d'habitat dans le secteur du bras supérieur (enrochements en hiver et ripisylve absente en été). Ce résultat confirme celui de Grossman et al. (1987). Dans le milieu non perturbé (bras supérieur), les interstices entre les blocs d'enrochement offrent des caches aux poissons. En été, le toxostome change pour des macrohabitats sans ripisylve mais à végétation aquatique riche en nourriture et abritée du courant. Contrairement à son comportement 
dans le bras non perturbé, le toxostome se répartit indifféremment dans l'ensemble des habitats du bras inférieur. L'hypothèse d'un effet stimulant de la température (et/ou de la pollution) sur la mobilité des poissons peut être retenue. Lobon-Cervia \& De Diego (1988) ont eux aussi noté une redistribution des espèces vers les zones plus profondes, où elles se maintiennent pendant l'hiver.

La perche donne un autre exemple de choix actif d'habitat, en été, entre le bras supérieur (berges enrochées ou nues) et le bras inférieur (berges recouvertes d'arbres clairsemés). L'absence de ripisylve allant de pair avec une végétation aquatique riche en macroinvertébrés dont se nourrit la perche (Diehl 1993), le choix d'habitat peut être justifié par le facteur trophique.

L'effet de la pollution ammoniacale avec des teneurs non négligeables et chroniques semble avoir très peu d'influence sur la stratégie d'occupation des macrohabitats. En effet, le facteur thermique est suffisant pour expliquer des singularités de comportement des poissons vis-à-vis de l'occupation des habitats du bras inférieur.

Dans le cas des secteurs 2 (pseudo-naturel) et 3 (rejets industriels), il serait intéressant d'étudier l'effet thermique sur la structure du peuplement et la mobilité spatio-temporelle afin d'analyser plus finement les relations poisson-habitat.

\section{Remerciements}

Les débits de la Garonne à l'entrée de Toulouse ont été aimablement fournis par la DDE (Service Hydrologique) ainsi que les mesures de qualité de l'eau par l'Agence Adour-Garonne. Les données sur les rejets industriels de l'agglomération ont été communiquées par la DRIRE Midi-Pyrénées. Les auteurs remercient également la Fédération des Associations agréés pour la Pêche et la Protection des Milieux Aquatiques en Haute-Garonne, pour les facilités et l'aide accordées. Ce travail a été réalisé dans le cadre du programme GIS-ECOBAG.

\section{Travaux cités}

Alatalo R. V. 1981. - Problems in the measurement of evenness in ecology. Oikos, 37 (2) : 199-204.

Baras E. \& Cherry B. 1990. - Seasonal activities of female barbel (Barbus barbus, L.) in the river Ourthe as revealed by radio tracking. Aquatic Living Resources, 3 : 283-294.

Belaud A., Bengen D. \& Lim P. 1989. - Approche de la structure du peuplement ichtyologique de six bras morts de la Garonne. Annls Limnol., 26 (1): 81-90.

Bengen D. 1992. - Contribution à la typologie fonctionnelle des bras morts de la Garonne : Approches physico-chimique et ichtyologique. Thèse de Doctorat, INP Toulouse. $124 \mathrm{p}$.

Bengen D., Belaud A. \& Lim P. 1992. - Structure et typologie ichtyenne de trois bras morts de la Garonne. Annls Limnol., 28 (1): $35-56$.
Bouchard P. 1996. - Dynamique spatio-temporelle des peuplements ichtyologiques de la Durance aménagée. Thèse de Doctorat, Université de Provence : $223 \mathrm{p}$.

Braband A. 1985. — Food of roach and ide. Oecologia, 66 : 461467.

Changeux T. 1994. - Structure des peuplements de poissons a l'échelle du bassin rhodanien. Approche régionale et organisation longitudinale. Exploitation des captures par pêche aux engins. Thèse de Doctorat, Université de Lyon I : 241. p.

Chappaz R. 1986. - Etude piscicole de la retenue de Sainte Croix. Fontaine l'Evêque. Thèse de Doctorat, Université de Provence, Aix - Marseille I : 193 p.

Connell J. H. 1978. - Diversity in tropical rainforests and coral reefs. Sciences, $199: 1302-1310$.

Copp G. H. 1992. - An empirical model for predicting microhabitat of $0+$ juvenile fishes in a lowland river catchement. Oecologia (Berlin), 91 : 338-345.

Cross D. G. 1976. - A method of comparing the efficiencies of electric fishing operations. J. Fish. Biol., $9: 261-265$.

Daget J. 1976. - Les modèles mathématiques en écologie. Collection d'Ecologie ${ }^{\circ} 8$, Masson ed., Paris : 172 p.

Décamps H. \& Naiman R. J. 1989. - L'écologie des fleuves. La Recherche, $208: 310-319$.

Degiorgi F. 1993. - Etude de l'organisation spatiale de l'ichtyofaune lacustre. Thèse de Doctorat, Université de Franche-Comté, Besançon : $207 \mathrm{p}$.

Delmastro G. B. \& Balma G. A. C. 1990. - Primo reperto per l'Italia e acclimatazione del ciprinidae endemico balcanico Pachychilon pictus (Heckel \& Kner, 1858) nel fiume Serchio in Toscana (Osteichthyes : Cyprinidae). Estratto dal Bollettino del Museo Regionale di Scienze Naturali - Torino, 8 (2) : 411-416.

Diehl S. 1993. - Effect of habitat structure on resource availability, diet and growth of benthivorous perch, Perca fluviatilis. Oikos, $67: 403-414$.

Dolédec S. \& Chessel D. 1991. - Recent developments in linear ordination methods for environmental sciences. In Council of Scientific Research Integration ed. Trends in Ecology. Research Trends publishers, India.

Emerson K., Russo R. C., Lund R. E. \& Thurston R. V. 1975. Aqueous ammonia equilibrium calculations : Effect of $\mathrm{pH}$ and temperature. J. Fish. Res. Bd. Can, $32:$ 2379-2383.

Gisclard F. 1984. - Pollutions du bras inférieur de la Garonne à Toulouse. Impact général et cas d'un rejet acide. Thèse de Doctorat, INP Toulouse : $235 \mathrm{p}$.

Grossman G. D., Sostoa A., Freeman M. C. \& Lobon-Cervia J. 1987. - Microhabitat use in a mediterranean riverine fish assemblage. Fishes of the upper Matarraña. Oecologia 73 : 501-512.

Huet M. 1949. - Aperçu des relations entre la pente et les populations piscicoles des eaux courantes. Schweizerische Zeitschrift für Hydrologie, 11 (3) : 332-351.

Laurec A., Le Guen J. C. \& Frontier S. 1983. - Collecte de l'information pour une gestion rationnelle des stocks halieutiques, In : Frontiers S. (ed.), Stratégie d'échantillonnage en écologie. Masson, Paris : $385-415$.

Legendre L. \& Legendre P. 1979. - Ecologie numérique. Tome 2 : la structure des données écologiques. Masson, Paris et Presse de l'Université du Québec, $254 \mathrm{p}$.

Lelek A. \& Köhler C. 1989. - Zustandsanalyse der Fischartengemeinschaften im Rhein (1987-1988). Fischökologie, 1 : 47-64.

Lim P., Belaud A. \& Hutagalung R. 1994. - Etude hydrobiologique de la Garonne dans le périmetre de la concession de la Régie Municipale d'Electricité de Toulouse. En vue de la construction d'une micro-centrale hydroélectrique sur le barrage de la Cavaletade. Rapport INP-ENSAT/RMET : 113 p. 
Lim P., Belaud A. \& Labat R. 1985. - Peuplement piscicole de la Garonne entre St. Gaudens et Agen. Ichtyophysiologica Acta, $9: 187-201$.

Lobon-Cervià J. \& De Diego A. 1988. - Feeding strategy of the barbel (Barbus bocagei Steind.) with relation to the benthos composition. Arch. Hydrobiol., $114: 83-95$.

Mann R. H. K. 1996. - Environmental requirements of European non-salmonid fish in rivers. Hydrobiologia, 323: 223-235.

Michel P. \& Oberdorff T. 1995. - Feeding habits of fourteen European freshwater fish species. Cybium 19 (1) : 5-46

Nelva A. 1985. - Biogéographie, démographie et écologie de Chondrostoma nasus nasus (L., 1758) (Hotu, Poisson, Téléostéen, Cyprinidé). Thèse de Doctorat, Université $\mathrm{Cl}$. Bernard, Lyon I : $365 \mathrm{p}$.

Nelva A., Persat H. \& Chessel D. 1979. - Une nouvelle méthode d'étude des peuplements ichtyologiques dans les grands cours d'eau par échantillonnage ponctuel d'abondance. C. R Acad. Sci. Paris, 289 Série D : 1295-1298.

Oberdorff T., Guilbert E. \& Lucchetta J. C. 1993. - Pattern of fish species richness in the Seine River basin, France. Hydrobiologia, $259: 157-167$.

Oberdorff T. \& Hugues R. M. 1992. - Modification of an index of biotic integrity based on fishes assemblages to characterize rivers of the Seine Basin, France. Hydrobiologia, $228: 117-130$

Persat H., Nelva A. \& Chessel D. 1985. - Approche par l'analyse discriminante sur variables qualitatives d'un milieu lotique : le Haut-Rhîne français. Oecol. Gener, $6:$ 365-381.

Persat H., Nelva A., Pattee E., Perrin J. F. \& Roux A. L. 1981. Composition des peuplements piscicoles dans trois secteurs du Haut-Rhône : relations avec les paramètres du milieu. Actes du XXVI Cong. Nat. Orléans, Les grandes fleuves français : 145153.

Philippart J. C. \& Baras E. 1989. - The biology and management of the barbel in the Belgian River Meuse, with special reference to reconstruction of populations using intensively-reared fish. Proceedings of the $19^{\text {th }}$ Institut of Fisheries Management Annual Study Cource. Southampton, September 5-8, $1988: 61-82$.

Philippart J. C. 1977. - Contribution à l'hydrobiologie de l'Ourthe. Dynamique des populations et production de quatre espèces de poissons Cyprinidae : Barbus barbus (L.), Leuciscus cephalus (L.), Chondrostoma nasus (L.) et Leuciscus leuciscus (L.). Thèse de Doctorat, Université de Liège : $225 \mathrm{p}$.

Philippart J. C., Baras E. \& Rimbaud G. 1990. - Premières observations sur une population de juvéniles de saumons atlantiques (Salmo salar L.) reconstituées dans l'Ourthe en 1989. Cah. Ethol. Appl., 10 (3-4) : 421-440.
Poizat G. 1993. - Echelle d'observation et variabilité des abondances des juvéniles de poissons dans un secteur aval du Rhône. Thèse de Doctorat. Université Cl. Bernard, Lyon I : 206 p.

Pouilly M. 1994. - Relations entre l'habitat physique et les poissons des zones à cyprindés rhéophiles dans trois cours d'eau du bassin Rhodanien : vers une simulation de la capacité d'accueil pour les peuplements. Thèse de Doctorat. Université $\mathrm{Cl}$. Bernard, Lyon I : 256 p.

Ramade F. 1981. - Ecologie des ressources naturelles. Masson ed., Paris : 322 p.

Schiemer F. \& Zalewski M. 1992. - The importance of riparian ecotones for diversity and productivity of riverine fish communities. Netherlands journal of Zoology, 42 (2-3) : 323-335.

Steedman R. J. 1988. - Modification and assessment of an index of biotic integrity to quantify stream quality in southern Ontario. $\mathrm{Ca}$ nadian Journal of Fisheries and Aquatic Sciences, 45 : 492-501.

Stephanidis A. 1974. - On some fish of the Ionioko Rinthian region (W. Greece Ect.) - A new genus of Cyprinidae : Tropidophoxinellus N. Gen. - Biologia Gallo-Hallonica, 5 (2) : 235-257.

Swingle H.S. 1950. - Relationships and dynamics of balanced and unbalanced fish populations. Agric. Exp. Sta. Albana Polyt. Inst. Auburn. Bull. $n^{\circ} 274$.

Thioulouse J., Chessel D., Dolédec S. \& Olivier J.M. (sous-presse). - ADE-4 : a multivariate analysis and graphical display software. Statistics and Computing.

Thioulouse J., Dolédec S., Chessel D. \& Olivier J.M. 1994. — ADE software : multivariate analysis and graphical display of environmental data. In : Software per l'ambiente. Guariso G. \& Rizzoli A., ed. pp. 57-62, Patron Editore, Bologna.

Vannote R. L., Minshall G. W., Cummins K. W., Sedell J. R. \& Cushing C. E. 1980. - The river continuum concept. Can. J Fish. Aquat. Sci., $37: 130-137$.

Verneaux J. 1981. - Les poissons et la qualité des cours d'eau. Ann. Sci. Univ. de Franche-Comté, Besançon, $4^{\circ}$ série 2 : 33-41.

Vibert R. 1968. - Application de l'électricité à la biologie et à l'aménagement des pêches continentales. Acte Symp. FAO, Belgrade, Ed. INRA, Paris, 276 p.

Ward J. V. \& Stanford J. A. 1983. - The serial discontinuity concept of lotic ecosystems. In : T. D. Fontaine \& S. M. Bartell (ed.), Dynamics of lotic ecosystems. Ann. Arbor Science Publisher Inc., Ann Arbor. ML : 29-42.

Welcomme R. L. 1985. - River fisheries. FAO Fish. Tech. Paper, 262-330. 